\title{
A design strategy for phase synchronization in Precoding-enabled DVB-S2X user terminals
}

\author{
$1^{\text {st }}$ Liz Martínez Marrero \\ SIGCOM Research Group \\ SnT, University of Luxembourg \\ Luxembourg, Luxembourg \\ liz.martinez-marrero@uni.lu
}

\author{
$2^{\text {nd }} J u a n$ Carlos Merlano Duncan \\ SIGCOM Research Group \\ SnT, University of Luxembourg \\ Luxembourg, Luxembourg \\ juan.duncan@uni.lu
}

\author{
$3^{\text {rd }}$ Jorge Querol \\ SIGCOM Research Group \\ SnT, University of Luxembourg \\ Luxembourg, Luxembourg \\ jorge.querol@uni.lu
}

\begin{abstract}
$4^{\text {th }}$ Symeon Chatzinotas
SIGCOM Research Group

Luxembourg, Luxembourg

Symeon.Chatzinotas@uni.lu
\end{abstract}

$5^{\text {th }}$ Adriano Camps

Dept. of Signal Theory and Communications $6^{\text {th }}$ Björn Ottersten SIGCOM Research Group

Universitat Politècnica de Catalunya and IEEC/CTE-UPC SnT, University of Luxembourg

Barcelona, Spain

camps@tsc.upc.edu
Luxembourg, Luxembourg

bjorn.ottersten@uni.lu

\begin{abstract}
This paper address the design of a phase tracking block for the DVB-S2X user terminals in a satellite precoding system. The spectral characteristics of the phase noise introduced by the oscillator, the channel, and the thermal noise at the receiver are taken into account. Using the expected phase noise mask, the optimal parameters for a second-order PLL intended to track channel variations from the pilots are calculated. To validate the results a Simulink model was implemented considering the characteristics of the hardware prototype. The performance of the design was evaluated in terms of the accuracy and stability for the frame structure of superframe Format 2, as described in Annex E of DVB-S2X.

Index Terms-PLL design, phase tracking, DVB-S2X standard, phase noise, satellite precoding, CSI estimation
\end{abstract}

\section{INTRODUCTION}

The second-generation satellite digital video broadcasting standard (DVB-S2) and its extension (DVB-S2X) introduced new modulation and coding pairs, resulting in finer signal-tonoise ratio (SNR) threshold granularity, and a wider range of SNR than the original DVB standard. Although DVBS2X supports very high order modulation schemes (up to 256 APSK) [1] its performance is more sensitive to phase noise and non-linear distortion for these cases. Besides, emerging techniques such as satellite precoding [2] and beam-hopping transmission [3] designed to be compatible with the SuperFrame (SF) Formats of DVB-S2X require accurate carrier synchronization and estimation algorithms.

Specifically, Precoding technique aims at reducing the interbeam interference, for which it requires the knowledge of the Channel State Information (CSI) for each of the signals that have to be precoded [4]. The user terminals estimate the CSI for each of the received waveforms and feedback this information to the gateway. To this aim, the terminals lock on each waveform following the synchronization procedure defined in the annex C.5 of the DVB-S2X standard [5]. This procedure consists of the next sequence of tasks performed

This work was supported by the Fond National de la Recherche Luxembourg, under the CORE project COHESAT: Cognitive Cohesive Networks of Distributed Units for Active and Passive Space Applications and the Bridges Program DISBuS: Dynamic Beam Forming and In-band Signalling for Next Generation Satellite Systems. at each user terminal (UT): 1) coarse frequency estimation by means of a non-data aided estimator on the received waveform; 2) frame synchronization; 3) time tracking for each waveform; 4) fine phase and frequency tracking; and 5) channel estimation.

Among these tasks, the fine phase synchronization has been extensively studied since the phase noise limits the accuracy of the channel estimator. The conventional carrier phase recovery is based on a Phase Locked Loop (PLL) with feedback loop, feedforward estimator or estimator by linear interpolation between two consecutive pilot symbol fields. For example, [6] proposed an architecture for DVB-S2 receivers where the phase synchronization is performed in two steps separated by the automatic gain control. First, the Coarse Phase Synchronization block applies Maximum-Likelihood (ML) estimation and a linear interpolation method to estimate the phase on one pilot field. It removes the phase ambiguity for constellation demapping, achieving near perfect recovery performance for QPSK and 8PSK. However, for high-order and more phase jitter sensitive modulations, additional fine phase synchronization is needed. The Fine Phase synchronization employs a non decoder-aided phase synchronization technique based on a digital PLL [6]. Another design based on a PLL structure was proposed in [7]. In this case the filter gain and scaling factor of the loop were set after consideration of circuit stabilities and complexities. According to [8], it is not feasible to do phase tracking with a PLL for the DVB-S2X at very low SNR, which increses phase lock loss. In this case, the authors proposed to estimate the residual frequency offset and phase noise using the phase offset difference between consecutive pilot fields.

However, the standard suggests using a PLL for frequency and phase tracking before the channel estimation [5]. In this case, the channel estimation is performed on the distributed pilots of the superframe, which are conformed by BPSK symbols. The design of a PLL depends on the spectrum of the intended signal to be tracked [9], and in this specific case the phase tracking block has to cope with a residual carrier frequency error from the previous blocks and with a strong phase noise [5]. According to the channel model in ETSI TR 
102 376-1 [5], the maximum carrier frequency instability is $\pm 5 \mathrm{MHz}$. Besides, the phase noise mask takes into account the contributions from the uplink, the satellite payload, and the UT [5]. Among these components the most critical values are from the phase noise of the UT's oscillator. Even when this term has no influence on the channel estimation accuracy by itself, it can impose a significant error source for the phase synchronization which indirectly affects the CSI estimation.

Some previous works related to the design of the DVB-S2 UT's demodulators consider closed synchronization loops for fine phase tracking. For instance, [10] and [11] analized in details the pilot aided phase estimation design for DVB-S2 demodulators. In these articles, the authors refered to the need of the phase recovery block to cope with residual frequency offset resulting from previous carrier recovery procedures. Most of these residual frequency offset can be related to the phase noise PSD, but the authors didn't consider it in the design.

This paper, focuses on the design of the "fine phase synchronization" block of DVB-S2X UTs taking into account the PSD of different error sources, which will be detailed in Section II. After the system model description in Section III, Section IV provides an insight into the optimal design of the PLL's parameters. Results and conclusions are presented in sections V and VI, respectively.

Among the main contributions of this article are: 1) the design of a PLL to track the phase variations, previous to the CSI estimation, in the DVB-S2X UT; 2) considering the spectral characteristic of the phase noise introduced by the oscillator, the channel, and the UT's thermal noise for the design; 3) proposing a design strategy to calculate the optimal PLL parameters based on the phase noise mask of the oscillators at the satellites.

\section{ERROR SOURCES FOR SYNCHRONIZATION}

The tracking performance of a PLL is affected by the influence of several error sources. In addition to the thermal noise and dynamic stress error, oscillator phase noise can cause significant phase jitter which degrades the tracking performance [12].

\section{A. Oscillator phase noise}

Oscillator phase noise can be either natural phase noise caused by the oscillator itself or "external" phase noise caused by mechanical vibrations [12]. Quartz crystal oscillators change frequency slightly when accelerated. Crystals exhibit an acceleration sensitivity, which means that the random and periodic mechanical vibrations found in many types of equipment can induce significant phase noise in high-performance crystal oscillators. Generally, higher frequency crystals will have less g-sensitivity. Besides, physical mounting techniques can absorb the occurring vibrations to some extent. In the context of this paper, deployed satellites and static receivers are considered, so the effects of the vibration phase noise can be neglected in our analysis [12]. Therefore, this study only considers the physical behavior of the oscillators as the source of the phase noise of the system.
Empirical models suggest that the phase noise power spectral density (PSD), $S_{\phi}(f)$, can be described as a sum of power-law processes [13]:

$$
S_{\phi}(f)= \begin{cases}\sum_{\alpha=-4}^{0} h_{\alpha} f^{\alpha} & 0<f<f_{h} \\ 0 & f \geq f_{h}\end{cases}
$$

where $f_{h}$ is the high-frequency cut-off of an ideal (i.e. infinitely sharp) low-pass filter [14]. The $h_{\alpha} f^{\alpha}$ terms are related to random walk frequency modulated (FM) $(\alpha=-4)$, flicker FM $(\alpha=-3)$, white FM $(\alpha=-2)$, flicker $(\alpha=-1)$, and white phase noise $(\alpha=0)$ respectively [15].

According to the theoretical model described in (1), the random walk FM noise term, $h_{-4} f^{-4}$, continues increasing infinitely while frequency approaches oscillator's nominal value. However, more recent studies consider an additional Gaussian segment [15] for frequencies closer to the nominal value. This model includes the frequency drifts of a practical and realistic system, which is similar to a frequency modulation or spreading of the main carrier.

\section{B. Thermal Noise}

Thermal noise in all electronic devices results from the random motion of free electrons in a conductor due to thermal agitation. Therefore, the thermal noise PSD, $S_{N}(f)$, is directly proportional to the system noise temperature $T$,

$$
S_{N}(f)=N_{0}=k T
$$

where $k=1.38 \times 10^{-23} \mathrm{~J} / \mathrm{K}$ is Boltzmann's constant.

\section{SySTEM MODEL}

The system model considered in this work consists of a geostationary satellite transmitting to two DVB-S2X receivers on the ground, in different beams as shown in Fig. 1. The beams are generated using two independent oscillators. This is a feasible assumption in practical satellite systems due to technical constraints, such as independency between payloads, autonomy, robustness, cross-interference between RF channels and redundancy, where the whole system should not rely on the same oscillator [16]. The model employs Precoding technique to avoid inter-beam interference.

The bundled frame structure of superframe format 2, as described in Annex E of DVB-S2X, includes 9 bundled payload frames at each superframe. Each bundled frame contains a header, a precoded pilot field, 71 non-precoded pilots, and 64800 payload symbols [17]. The pilot fields include 36 BPSK symbols separated from each other by 956 payload symbols. The superframe format 2 of the DVB-S2X standard is represented in Fig. 2. The phases of the symbols from each pilot are used at the UT to estimate the CSI.

To analyze the effects of the phase noise on the channel estimation at the UTs, we consider the transmitted pilot vector $\mathbf{x}(t)=\left[\begin{array}{ll}x_{1}(t) & x_{2}(t)\end{array}\right]^{\prime}$, where $x_{i}(t)=s_{i}(t) \mathrm{e}^{j \phi_{i}(t)}$ with $i=\{1,2\}$. The signal $s_{i}(t)$ is a BPSK modulated Walsh Hadamard sequence and $\mathrm{e}^{j \phi_{i}(t)}$ is the phase noise introduced by the LO of the satellite in the beam $i$. The uplink signal is considered ideal since the phase noise introduced by the GW affects in the same way $s_{1}(t)$ and $s_{2}(t)$, and it can be considered as part of the channel distortion. 


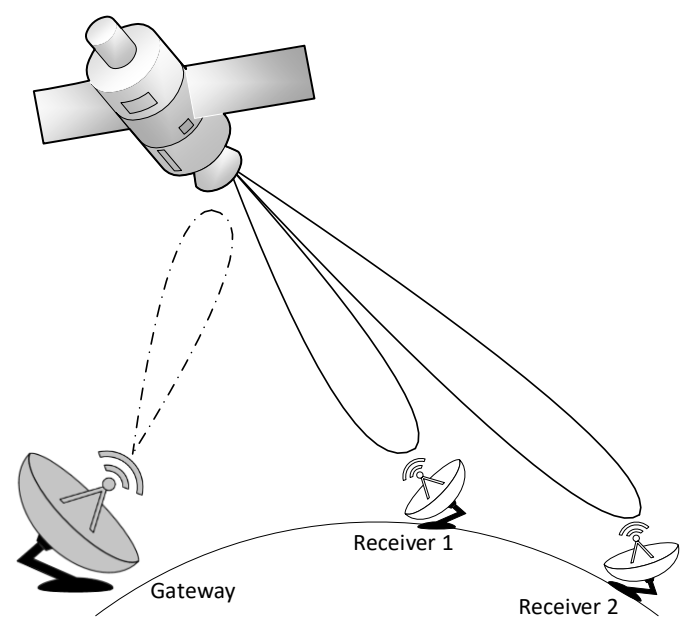

Figure 1: System model: One satellite transmitting to two DVB-S2X UTs through two beams generated by independent LOs.

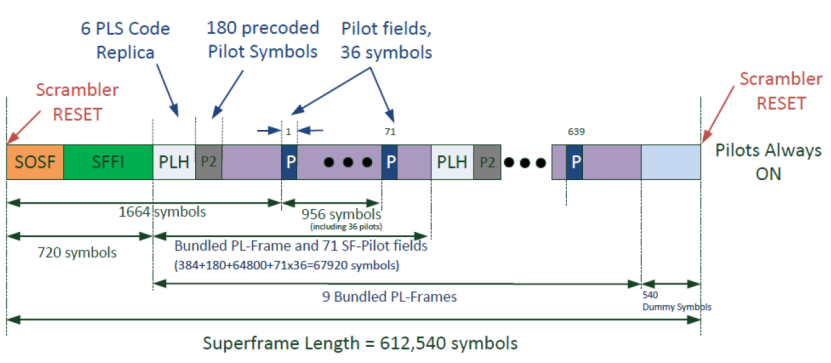

Figure 2: Format 2 of the superframe structure from the DVB-S2x standard [17].

For these transmitted signals, the received signal vector is: $\mathbf{r}(t)=\mathbf{H}^{T} \mathbf{x}(t)+\mathbf{z}(t)$, where $\mathbf{H} \in \mathbb{C}^{2 \times 2}, H=\left[\begin{array}{ll}h_{11} & h_{12} \\ h_{21} & h_{22}\end{array}\right]$ is the channel matrix and $\mathbf{z}(t) \in \mathbb{C}^{2 \times 1}$ is the AWGN at each receiver.

Considering the UT's phase noise as independent phase rotations $\theta_{r} \in \mathbb{R},-\pi<\theta_{r}<\pi$, the inputs of the PLLs are:

$$
\begin{aligned}
p_{1}(t)= & h_{11} s_{1}(t) \mathrm{e}^{j\left(\phi_{1}(t)+\theta_{1}(t)\right)}+ \\
& h_{12} s_{2}(t) \mathrm{e}^{j\left(\phi_{2}(t)+\theta_{1}(t)\right)}+z_{1}(t) \mathrm{e}^{j \theta_{1}(t)} \\
p_{2}(t)= & h_{21} s_{1}(t) \mathrm{e}^{j\left(\phi_{1}(t)+\theta_{2}(t)\right)}+ \\
& h_{22} s_{2}(t) \mathrm{e}^{j\left(\phi_{2}(t)+\theta_{2}(t)\right)}+z_{2}(t) \mathrm{e}^{j \theta_{2}(t)}
\end{aligned}
$$

Note that the terms $h_{i j} \mathrm{e}^{j \phi_{j}(t)}$ in (3) are the CSI to be estimated. However, the phase noise from the receiver's $\mathrm{LO} \mathrm{e}^{j \theta_{i}(t)}$, is common to both beams at each receiver and it introduces erros in the CSI estimation. For this reason, the fine phase synchronization mentioned in the DVB-S2X standard has to be performed in two steps, a first one to mitigate the phase noise from the receiver followed by a Digital PLL (DPLL) to track $h_{i j} \mathrm{e}^{j \phi_{j}(t)}$.

The Simulink model of DPLL to track these signals is represented in Fig. 3. It is a traditional second-order DPLL with slight variations in the phase detector to take advantage of the superframe format. The blocks inside the dashed box average the phases from each pilot to make the phase detection more robust. A Symbols counter block has also been included to account for the separation between consecutive pilots. Besides, the delay of the real system is included.

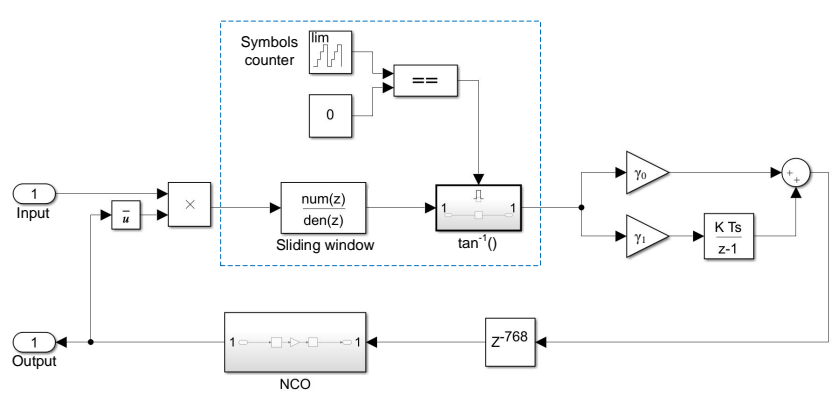

Figure 3: Simulink model of the second-order DPLL to track the phase of the pilots the Format 2 of the DVB-S2X standard.

\section{PLL DESIGN}

The DPLL in Fig. 3 is approximated by its analog equivalent, represented in Fig. 4. According to [18], this approximation is accurate when the loop noise bandwidth is smaller than the symbol rate, as it is in this case study. The input and estimated phases are $\theta_{i}$ and $\theta_{o}$ respectively. In steady-state conditions $\theta_{o}$ fluctuates around $\theta_{i}$ in magnitudes small enough to consider $D\left(\theta_{i}-\theta_{o}\right) \approx\left(\theta_{i}-\theta_{o}\right)$ [18]. The input $\theta_{n}$ represents the additive thermal noise on the receiver side combined with the channel noise (AWGN). The phase average for each pilot, represented in dashed lines in Fig. 3, was included in the model as part of the PSD of $\theta_{n}$.

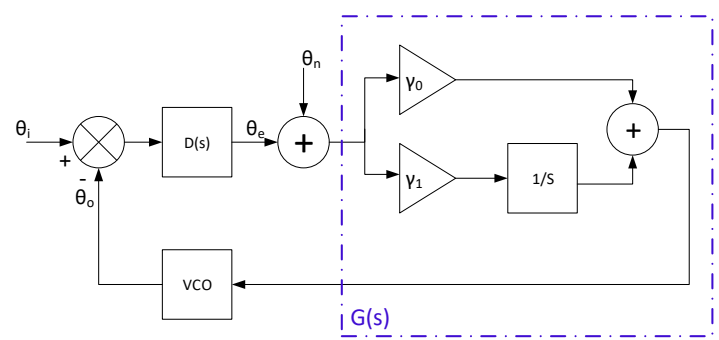

Figure 4: Equivalent continuous version of the DPLL in Fig. 3.

The basic loop equation for the PLL represented in Fig. 4 are:

$$
H(s)=\frac{\theta_{o}}{\theta_{i}}=\frac{G(s)}{1+G(s)}=1-E(s),
$$

where $G(s)=\frac{\gamma_{0}+\frac{\gamma_{1}}{s}}{s}$ and $H(s)$ are the open-loop and closedloop transfer functions, and $E(s)$ is the error response of the loop [9].

Equation (4) shows that the PLL has a restricted bandwidth. The loop tracks the input phase modulation within the loop bandwidth, and fails to track the phase modulation outside the bandwidth. The error response is necessarily complementary: the input phase modulation within the loop bandwidth is tracked with small error, whereas the input modulation 
outside the loop bandwidth is hardly tracked at all, resulting in almost $100 \%$ tracking error. Then, the phase noise power, $\sigma_{\theta}^{2}$, can be calculated as the spectrum of untracked phase jitter integrated over all frequencies.

$$
\sigma_{\theta}^{2}=\int_{0}^{\infty} S_{\phi}(f)\left|E_{\phi}(f)\right|^{2} d f+\int_{0}^{\infty} S_{N}(f)\left|E_{N}(f)\right|^{2} d f,
$$

where $S_{\phi}(f)$ is the oscillator noise PSD analyzed in Section II, and $S_{N}(f)$ is the PSD of the additive thermal noise on the receiver side combined with the channel noise. This is represented as the input $\theta_{n}$ in Fig. 4. The terms $|E(f)|$ are the amplitude of the error responses of the loop, and it can be obtained evaluating $s=j 2 \pi f$ in $E_{\phi}(s)=\frac{\theta_{e}}{\theta_{i}}=\frac{1}{1+G(s)}$ and $E_{N}(s)=\frac{\theta_{e}}{\theta_{n}}=-\frac{G(s)}{1+G(s)}$.

For the sake of simplicity, the phase noise power due to AWGN $\sigma_{\theta_{N}}^{2}$, and the oscillator phase noise $\sigma_{\theta_{\phi}}^{2}$ are calculated independently. The natural frequency $\omega_{n}$, and the damping factor $\zeta$ is a convenient description of the properties of a pole pair and so are often used to analyze second-order PLL loops. Besides, this notation is easily associated with the PLL bandwidth. Describing the Error Response as a function of $\omega_{n}$ and $\zeta, E_{\phi}(s)$ and $E_{N}(s)$ become:

$$
E_{\phi}(s)=\frac{s^{2}}{s^{2}+\gamma_{0} s+\gamma_{1}}=\frac{s^{2}}{s^{2}+2 \zeta \omega_{n} s+\omega_{n}^{2}},
$$

and

$$
E_{N}(s)=-\frac{\gamma_{0} s+\gamma_{1}}{s^{2}+\gamma_{0} s+\gamma_{1}}=-\frac{2 \zeta \omega_{n} s+\omega_{n}^{2}}{s^{2}+2 \zeta \omega_{n} s+\omega_{n}^{2}},
$$

with $\omega_{n}=\sqrt{\gamma_{1}}$ and $\zeta=\frac{\gamma_{0}}{2} \frac{1}{\sqrt{\gamma_{1}}}$.

To simplify the equations, it is useful to express the Error Response in terms of $\zeta$ and $K=2 \zeta \omega_{n}$. Then, the square of the amplitude of the Error Responses are:

$$
\left|E_{\phi}(f)\right|^{2}=\frac{(4 \pi f \zeta)^{2}}{\left(8 \pi f \zeta^{2}\right)^{2}\left(K^{2}+4 \pi^{2} f^{2}\right)-2(4 \pi K f \zeta)^{2}+K^{4}}
$$

and

$$
\left|E_{N}(f)\right|^{2}=\frac{\left(4 \pi f \zeta^{2}\right)^{2}+K^{2}}{\left(8 \pi f \zeta^{2}\right)^{2}\left(1+4 \pi^{2} f^{2} / K^{2}\right)-2(4 \pi f \zeta)^{2}+K^{2}} .
$$

\section{A. Oscillator phase noise power}

The noise power due to the oscillator phase noise was calculated in [9] by multiplying (8) with $S_{\phi}(f)$ and solving the integral in $(5)$ :

$$
\begin{aligned}
\sigma_{\theta_{\phi}}^{2}= & h_{-4} \frac{16 \pi^{2} \zeta^{2}}{K^{3}}+h_{-3} \frac{4 \pi}{K^{2}}\left(1+2 \pi \zeta-\zeta^{2}\right) \\
& +h_{-2} \frac{\pi^{2}}{K}+\sigma_{\phi_{-1}}^{2}+\sigma_{\phi_{0}}^{2}
\end{aligned}
$$

According to [9], terms $\sigma_{\phi_{-1}}^{2}$ and $\sigma_{\phi_{0}}^{2}$ in (10) are too complex for display or straightforward understanding. Gardner proposes to use approximations considering a damping factor $\zeta \approx 1$, which is often employed for many PLLs [9]. Equations (11) to (13) show the approximated results for $\zeta \approx 1$, for an ideal low-pass (12) filter and a one-pole lowpass filter (13)

$$
\begin{aligned}
& \sigma_{\phi_{-1}}^{2} \approx h_{-1} {\left[\ln \left(\frac{4 \pi B}{K}\right)-1 / 2\right], \quad K<<4 \pi B } \\
& \sigma_{\phi_{0}}^{2} \approx h_{0} B, \quad K<<\frac{16 B}{3} \\
& \sigma_{\phi_{0}}^{2} \approx \frac{h_{0} \pi B}{2}, \quad K<<\pi B
\end{aligned}
$$

where $B$ is the noise bandwidth of an ideal low-pass filter and $\pi B / 2$ is the noise bandwidth of a one-pole lowpass filter. The inequalities specified for justification of the approximate results in (11) to (13) are applicable in most practical situations. Moreover, the ideal low-pass filter, (12), and the one-pole roll-off low-pass filter, (13), are extreme cases of low-pass filters; almost any other practical lowpass filter will have properties in between these two extremes, $h_{0} B \leq \sigma_{\phi_{0}}^{2} \leq \frac{\pi}{2} h_{0} B$ [9].

\section{B. Thermal noise power}

The noise power introduced by the channel and the receiver is calculated by:

$$
\sigma_{\theta_{N}}^{2}=\int_{0}^{\infty} S_{N}(f)\left|E_{N}(f)\right|^{2} d f
$$

Equations (2) and (9) are evaluated in (14), and the integral was solved using a computer-algebra program. The result is shown in (15)

$$
\begin{aligned}
& \sigma_{\theta_{N}}^{2}=\frac{N_{0} K\left(1+\zeta^{2}\right)}{16 \zeta^{2}}\left(\zeta-\sqrt{-1+\zeta^{2}}\right) \\
& \sqrt{-1+2 \zeta\left(\zeta+\sqrt{-1+\zeta^{2}}\right)}
\end{aligned}
$$

Similarly to the previous section, an approximation considering the damping factor $\zeta \approx 1$ can be made.

$$
\sigma_{\theta_{N}}^{2} \approx \frac{N_{0} K\left(1+\zeta^{2}\right)}{16 \zeta^{2}}
$$

\section{Optimization of the loop performance}

From (10) and (15), it is possible to design the PLL for the expected AWGN and phase noise characteristics of the system. To obtain the optimal values of $\gamma_{0}$ and $\gamma_{1}$, the minimization problem below has to be solved:

$$
\min _{\left(\zeta, \omega_{n}\right)}\left\{\sigma_{\theta_{\phi}}^{2}+\sigma_{\theta_{N}}^{2}\right\}
$$

This problem yields the nonlinear system of equations represented in (18). As it can be appreciated, the terms related to the different phase noises and the AWGN are completely independent. This allows to evaluate their inclusion in the equation according to the available phase noise characterization and the desired accuracy for the design.

$$
\left\{\begin{array}{l}
h_{-4} \frac{2 \pi^{4}}{\zeta^{2} \omega_{n}{ }^{3}}+h_{-3} \frac{2 \pi(1+\pi \zeta)}{\zeta^{3} \omega_{n}^{2}}+h_{-2} \frac{\pi^{2}}{2 \zeta^{2} \omega_{n}}+h_{-1} \frac{1}{\zeta}-N_{0} \frac{\omega_{n}\left(-1+\zeta^{2}\right)\left(\zeta+\sqrt{-1+\zeta^{2}}\right)}{8 \zeta^{2} \sqrt{-1+2 \zeta^{2}+2 \zeta \sqrt{-1+\zeta^{2}}}}=0 \\
h_{-4} \frac{6 \pi^{4}}{\zeta \omega_{n}{ }^{4}}+h_{-3} \frac{2 \pi\left(1+2 \pi \zeta-\zeta^{2}\right)}{\zeta^{2} \omega_{n}{ }^{3}}+h_{-2} \frac{\pi^{2}}{2 \zeta \omega_{n}{ }^{2}}+h_{-1} \frac{1}{\omega_{n}}-N_{0} \frac{\left(1+\zeta^{2}\right)\left(\zeta-\sqrt{-1+\zeta^{2}}\right) \sqrt{-1+2 \zeta\left(\zeta+\sqrt{-1+\zeta^{2}}\right)}}{8 \zeta}=0
\end{array}\right.
$$


The exact solutions of (18) cannot be determined by algebraic methods. In those cases, numerical methods should be used to approximate the solutions. In this paper, we used the Newton-Raphson method, which is one of the most popular numerical methods used in nonlinear systems [19].

\section{NUMERICAL RESULTS}

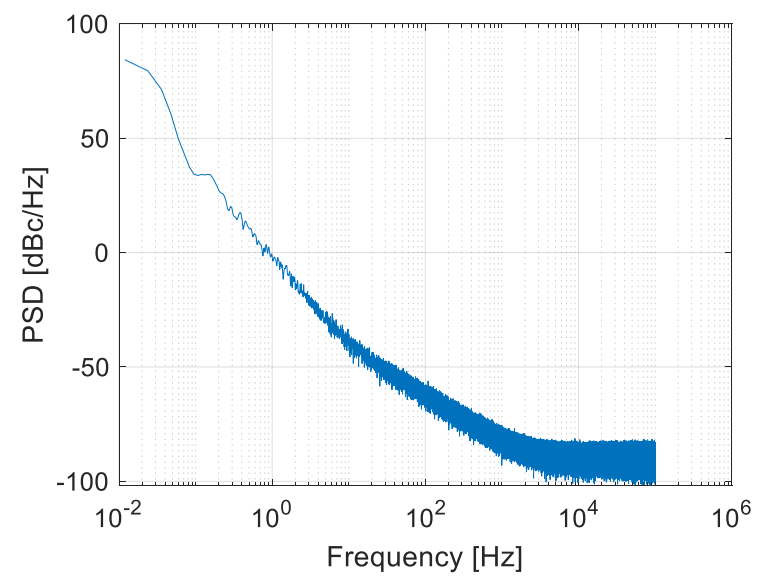

Figure 5: Phase noise PSD.

To validate our design we considered the Format 2 structure of the DVB-S2X standard and the phase noise mask shown in Fig. 5, whose parameters are $h_{-4}=70.2, h_{-2}=0.5$, $N_{0}=2.48 \times 10^{-5}$ and $h_{-3}=h_{-1}=h_{0}=0$. These values were selected according to the phase noise masks shown in Table I from the DVB-S2X standard [5].

Table I: Phase noise masks proposed by the DVB-S2X standard [5]

\begin{tabular}{|c|c|c|}
\hline \multirow{2}{*}{$\begin{array}{c}\text { Offset } \\
(\mathbf{H z})\end{array}$} & \multicolumn{2}{|c|}{ SSB Profile $^{\mathrm{a}}(\mathbf{d B c / H z})$} \\
\cline { 2 - 3 } & satellite & receivers \\
\hline 10 & & \\
\hline 100 & -62 & -25 \\
\hline $1 \mathrm{~K}$ & -80 & -50 \\
\hline $10 \mathrm{~K}$ & -90 & -73 \\
\hline $100 \mathrm{~K}$ & -95 & -93 \\
\hline $1 \mathrm{M}$ & -106 & -103 \\
\hline $10 \mathrm{M}$ & -116 & -114 \\
\hline $50 \mathrm{M}$ & -118 & -117 \\
\hline
\end{tabular}

Using the Newton-Raphson method to solve (18), we obtained the optimal PLL configuration for this phase noise mask: $\omega_{n}=257.6 \pm 1$ and $\zeta=3.74 \pm 0.01$. Figure 6 shows the noise power function $\sigma_{\theta}^{2}\left(\omega_{n}, \zeta\right)$ evaluated for these parameters.

To validate the accuracy of the simplification performed on the DPLL (Fig. 3), when it was approximated by its continuous equivalent (Fig. 4), the average system response of the DPLL is compared against the closed-loop transfer function of the simplified model, denoted as $H(s)$ in (4). This validation is represented in Fig. 7, where the red lines correspond to the amplitude and phase of $H(s)$, obtained by evaluating the optimal parameters $\omega_{n}$ and $\zeta$ in (4). The signal
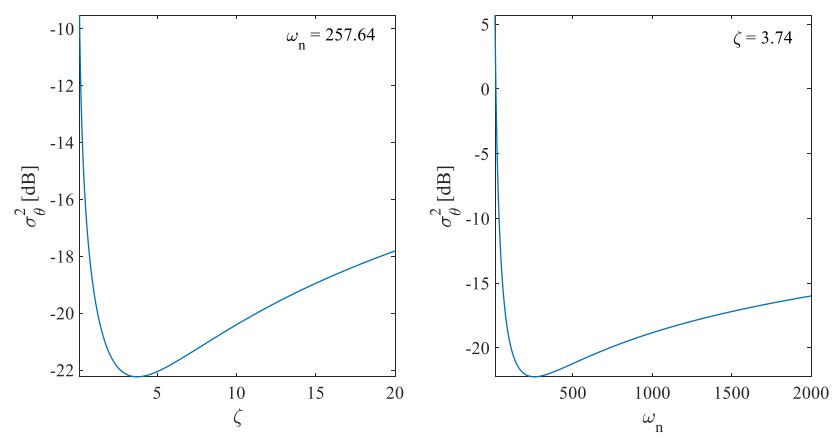

Figure 6: Noise power function $\sigma_{\theta}^{2}\left(\omega_{n}, \zeta\right)$ for the optimal parameters: a) $\sigma_{\theta}^{2}(257.6, \zeta)$ and b) $\sigma_{\theta}^{2}\left(\omega_{n}, 3.74\right)$.

represented in blue in Fig. 7 is the DPLL system response, obtained through Monte Carlo simulations with 150 iterations on the Simulink model shown in Fig. 3.
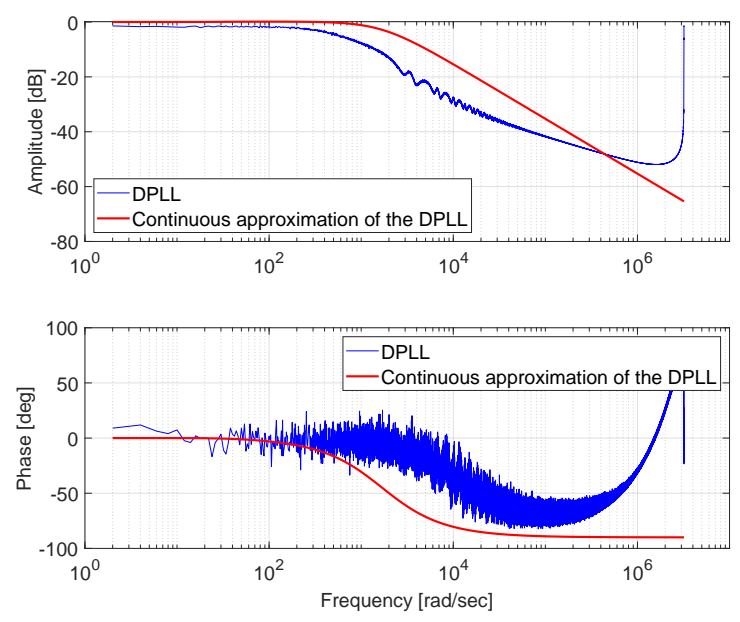

Figure 7: Bode Diagram of the closed-loop transfer function of the DPLL's contiuous approximation, $H(s)$ (red line) vs. DPLL system response (blue line).

As it can be noticed, there is a gap between the continuous approximation transfer function and the DPLL system response. This is mainly due to the simplification of the 32 symbols phase average for each pilot. As the optimal values were calculated for the continuous approximation, it is possible to decrease the gap between both system responses by changing the value of $\omega_{n}$. For this purpose, $\omega_{n}$ was iteratively increased keeping $\zeta=3.74$. Figure 8 shows some of the most significant results of this operation. As it can be appreciated in the figure, the system response approximates the simplified transfer function as $\omega_{n}$ increases. However, for $\omega_{n}>700$ the amplitude response at high frequencies is distorted. For this reason we selected $\omega_{n}=600$, which is slightly closer to the simplified model than the original curve, and keeps a similar aspect.

Once we selected the value of $\omega_{n}$ that best approximates to the simplified model, the phase error for different values of $\zeta$ was calculated. These results are included in Table II along with the results for other values of $\omega_{n}$ that could be used as validation. Considering the data in Table II, the lowest phase 
Table III: Characteristics of the designed PLL

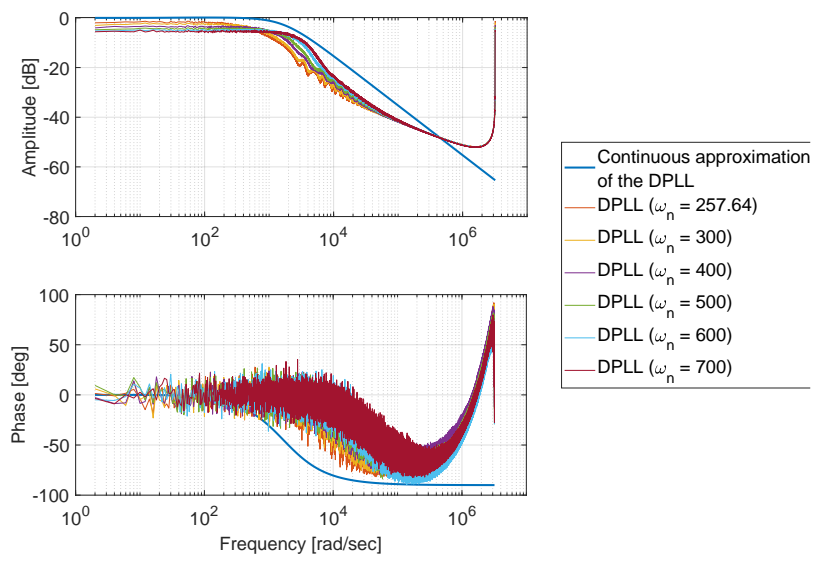

Figure 8: Bode Diagram of the DPLL system response for different values of $\omega_{n}$ with $\zeta=3.74$.

error is obtained for $\omega_{n}=600$ and $\zeta=0.9$. We used this pair $\left(\omega_{n}, \zeta\right)$ to analyze the performance of the PLL considering parameters such as the stability and the noise bandwidth.

Table II: Mean squared phase error at the output of the phase detector for different values of $\left(\omega_{n}, \zeta\right)$

\begin{tabular}{|c|c|c|c|c|c|}
\hline \multicolumn{2}{|c|}{} & \multicolumn{5}{|c|}{$\omega_{n}$} \\
\cline { 3 - 6 } \multicolumn{2}{c|}{} & $\mathbf{2 5 7 . 6 4}$ & $\mathbf{4 0 0}$ & $\mathbf{6 0 0}$ & $\mathbf{8 0 0}$ \\
\hline \multirow{4}{*}{} & $\mathbf{0 . 5}$ & $2.785 \mathrm{E}-3$ & $1.642 \mathrm{E}-3$ & $1.365 \mathrm{E}-3$ & $1.342 \mathrm{E}-3$ \\
\cline { 2 - 6 } & $\mathbf{0 . 6}$ & $2.482 \mathrm{E}-3$ & $1.541 \mathrm{E}-3$ & $1.325 \mathrm{E}-3$ & $1.321 \mathrm{E}-3$ \\
\cline { 2 - 6 } & $\mathbf{0 . 7}$ & $2.270 \mathrm{E}-3$ & $1.474 \mathrm{E}-3$ & $1.305 \mathrm{E}-3$ & $1.318 \mathrm{E}-3$ \\
\cline { 2 - 6 } & $\mathbf{0 . 8}$ & $2.114 \mathrm{E}-3$ & $1.428 \mathrm{E}-3$ & $1.296 \mathrm{E}-3$ & $1.326 \mathrm{E}-3$ \\
\cline { 2 - 6 } & $\mathbf{0 . 9}$ & $1.995 \mathrm{E}-3$ & $1.396 \mathrm{E}-3$ & $\mathbf{1 . 2 9 5 E}-3$ & $1.341 \mathrm{E}-3$ \\
\hline & $\mathbf{1 . 0}$ & $1.901 \mathrm{E}-3$ & $1.374 \mathrm{E}-3$ & $1.301 \mathrm{E}-3$ & $1.363 \mathrm{E}-3$ \\
\cline { 2 - 6 } $\boldsymbol{5}$ & $\mathbf{1 . 5}$ & $1.642 \mathrm{E}-3$ & $1.342 \mathrm{E}-3$ & $1.382 \mathrm{E}-3$ & $1.540 \mathrm{E}-3$ \\
\cline { 2 - 6 } & $\mathbf{2 . 0}$ & $1.538 \mathrm{E}-3$ & $1.377 \mathrm{E}-3$ & $1.526 \mathrm{E}-3$ & $1.832 \mathrm{E}-3$ \\
\cline { 2 - 6 } & $\mathbf{2 . 5}$ & $1.500 \mathrm{E}-3$ & $1.446 \mathrm{E}-3$ & $1.732 \mathrm{E}-3$ & $2.298 \mathrm{E}-3$ \\
\cline { 2 - 6 } & $\mathbf{3 . 7 4}$ & $1.531 \mathrm{E}-3$ & $1.739 \mathrm{E}-3$ & $2.710 \mathrm{E}-3$ & $6.700 \mathrm{E}-3$ \\
\cline { 2 - 6 } & $\mathbf{5 . 0}$ & $1.668 \mathrm{E}-3$ & $2.286 \mathrm{E}-3$ & $6.823 \mathrm{E}-3$ & 3.585 \\
\hline
\end{tabular}

The stability of the system was analyzed through the phase margin of the PLL. Generally, a Phase Margin of $60^{\circ}$ to $70^{\circ}$ is enough to guarantee stability, in the present design, the Phase Margin is $73.5^{\circ}$, which makes the PLL very stable.

Other parameters such as noise bandwidth, pull-out range, lock-in range, and lock-in time were also calculated. These results, and the equations used, are listed in Table III. Note that the PLL lock-in time is relatively high, which is a characteristic of overdamped PLLs. This could be a problem in wireless systems such as the IP-based ones, but not for the DVB-S2X standards, where the transmission can be considered uninterrupted in time. In the same way, the noise bandwidth is small, and the pull-out range is wide compared to typical values [20], which implies that the system presents high tracking capabilities.

The mean squared phase error (MSPE) at the output of the phase detector is $0.017^{\circ}$ and it is obtained after $0.15 \mathrm{~ms}$. The MSPE was calculated for 837 pilots, equivalent to a $25 \mathrm{~ms}$ transmission. This result was averaged over 100 iterations.

\section{CONCLUSIONS}

Phase tracking is a crucial function for the DBV-S2X UT since the achieved accuracy in this operation determines the

\begin{tabular}{|c|c|c|}
\hline Parameter & Value & Equation \\
\hline Noise bandwidth & $353.33 \mathrm{~Hz}$ & $\frac{\omega_{n}}{2}\left(\zeta+\frac{1}{4 \zeta}\right)$ \\
\hline Pull-out range & $2.05 \mathrm{kHz}$ & $1.8(\zeta+1) \omega_{n}$ \\
\hline Lock-in range & $1.08 \mathrm{kHz}$ & $2 \zeta \omega_{n}$ \\
\hline Lock-in time & $10.47 \mathrm{~ms}$ & $\frac{2 \pi}{\omega_{n}}$ \\
\hline
\end{tabular}

performance of satellite Precoding. In this study, the DPLL to track the phase variations in the channel previous to the CSI estimation has been designed. Unlike previously published works [10] and [11], this article addresses the design of the fine phase tracking block considering the spectral characteristic of the phase noise introduced by the oscillator, the channel, and the UT's thermal noise.

As a result, the design strategy to achieve accurate phase tracking was obtained and it was demonstrated through a case study. Although the phase noise mask considered was described by the $h_{-4} f^{-4}, h_{-2} f^{-2}$ and $N_{0}$, the methodology allows to include any term of the phase noise PSD. Similarly, the case study used the Format 2 but the proposed design strategy is general and it can be used for other superframe formats of the DVB-S2X standard.

Among the limitations of the present work, we can state that the simplified model used does not perfectly describe the system response. However, an iterative method to obtain a better approximation has been implemented, which leads to lower phase errors and it is very feasible in practical applications. Future works could approach this topic by including the symbol phase average and modeling the optimization problem in the digital domain. This would get more accurate results at the price of increasing the complexity of the solution. However, for practical purposes, the proposed design strategy achieve accurate solutions, as was validated in the case study, where the MSPE was in the order of $0.001^{\circ}$.

\section{REFERENCES}

[1] S. Cioni and A. Ginesi, "DVB-S2X physical layer performance results over realistic channel models," International Journal of Satellite Communications and Networking, vol. 34, no. 3, pp. 361-376, 2016.

[2] S. Andrenacci, S. Chatzinotas, A. Vanelli-Coralli, S. Cioni, A. Ginesi, and B. Ottersten, "Exploiting orthogonality in DVB-S2X through timing pre-compensation," 8th Advanced Satellite Multimedia Systems Conference and 14th Signal Processing for Space Communications Workshop, ASMS/SPSC 2016, no. 978, pp. 1-8, 2016.

[3] C. Rohde, D. Rainish, A. Freedman, and G. Lesthievent, "BeamHopping System Configuration and Terminal Synchronization Schemes," in ICSSC2019, Okinawa, Japa, 2019.

[4] O. Kodheli, E. Lagunas, N. Maturo, S. K. Sharma, B. Shankar, J. F. M. Montoya, J. C. M. Duncan, D. Spano, S. Chatzinotas, S. Kisseleff, J. Querol, L. Lei, T. X. Vu, and G. Goussetis, "Satellite Communications in the New Space Era: A Survey and Future Challenges," pp. 1-45, 2020. [Online]. Available: http://arxiv.org/abs/2002.08811

[5] ETSI, "ETSI TR 102 376-2: ”Digital Video Broadcasting (DVB); Implementation Guidelines for the Second Generation System for Broadcasting, Interactive Services, News Gathering and Other Broadband Satellite Applications; Part 2: S2 Extensions (DVB-S2X)"," Tech. Rep., 2015.

[6] L. Qi, C. Xiaojun, and Z. Zucheng, "FPGA design and implementation of carrier synchronization for DVB-S2 demodulators," ASICON 2007 2007 7th International Conference on ASIC Proceeding, pp. 846-849, 2007.

[7] H. Jeon and D. G. Oh, "NCR clock recovery method suitable for DVB$\mathrm{S} 2 / \mathrm{RCS} 2$ systems," International Conference on ICT Convergence, pp. 995-996, 2014 
[8] P. Kim and J.-g. Ryu, "Carrier Phase Recovery for DVB-S2x standard in VL SNR channel," in ICSSC2019, Okinawa, Japa, 2019, pp. 2-5.

[9] F. M. Gardner, Phaselock Techniques, 3rd ed. John Wiley \& Sons, Inc., Hoboken, NJ., 2005.

[10] E. Casini, R. De Gaudenzi, and A. Ginesi, "DVB-S2 modem algorithms design and performance over typical satellite channels," International Journal of Satellite Communications and Networking, vol. 22, no. 3, pp. 281-318, 2004

[11] P. Savvopoulos, N. Papandreou, and T. Antonakopoulos, "Architecture and DSP Implementation of a DVB-S2 Baseband Demodulator," in 12th Euromicro Conference on Digital System Design, Architectures, Methods and Tools, no. September. Patras, Greece: IEEE, 2009, pp. 441-448.

[12] M. Irsigler and B. Eissfeller, "PLL Tracking Performance in the Presence of Oscillator Phase Noise," GPS Solutions, vol. 5, no. 4, pp. 45-57, 2002.

[13] J. Rutman, "Characterization of Phase and Frequency Instabilities in Precision Frequency Sources: Fifteen Years of Progress.” Proceedings of the IEEE, vol. 66, no. 9, pp. 1048-1075, 1978.

[14] I. Standards, IEEE Std 1139-2008 (Revision of IEEE Std 1139-1999) IEEE Standard Definitions of Physical Quantities for Fundamental Frequency and Time Metrology-Random Instabilities, 2009, vol. 2008, no. February.

[15] A. Chorti and M. Brookes, "A spectral model for RF oscillators with power-law phase noise," IEEE Transactions on Circuits and Systems I: Regular Papers, vol. 53, no. 9, pp. 1989-1999, 2006.

[16] M. A. Vazquez, A. Perez-Neira, D. Christopoulos, S. Chatzinotas, B. Ottersten, P.-D. Arapoglou, A. Ginesi, and G. Tarocco, "Precoding in Multibeam Satellite Communications: Present and Future Challenges," IEEE Wireless Communications, vol. 23, no. December, pp. 88-95, 2016.

[17] ETSI, "Digital Video Broadcasting (DVB); Second generation framing structure, channel coding and modulation systems for Broadcasting, Interactive Services, News Gathering and other broadband satellite applications; Part 2: DV B-S2 Extensions (DVB-S2X)," pp. 1-139, 2014.

[18] U. Mengali and A. N. D'Andrea, Synchronization Techniques for Digital Receivers (Applications of Communications Theory) 1997. New York, NY: Plenum Press.

[19] Richard L. Burden and J. D. Faires, "Numerical Solutions of Nonlinear Systems of Equations," in Numerical Analysis, 9th ed. Thomson Brooks/Cole, 2005, pp. 629-670.

[20] M. P. Li, "PLL Jitter and Transfer Function Analysis," in Jitter, Noise, and Signal Integrity at High-Speed, 1st ed. Prentice Hall, 2007, ch. 8. 